\title{
BMJ Open Effectiveness of extrinsic feedback for management of non-specific low back pain: a systematic review protocol
}

\author{
Daniel Cury Ribeiro, ${ }^{1}$ Aleksandra Katarzyna Mącznik, ${ }^{1}$ Stephan Milosavljevic, ${ }^{2}$ \\ J Haxby Abbott ${ }^{3}$
}

To cite: Ribeiro DC, Mącznik AK, Milosavljevic S, et al. Effectiveness of extrinsic feedback for management of non-specific low back pain: a systematic review protocol. BMJ Open 2018;8:e021259. doi:10.1136/ bmjopen-2017-021259

- Prepublication history for this paper is available online. To view these files, please visit the journal online (http://dx.doi. org/10.1136/bmjopen-2017021259).

Received 23 December 2017 Revised 14 March 2018 Accepted 5 April 2018
Check for updates

${ }^{1}$ School of Physiotherapy, Centre for Health, Activity, and Rehabilitation Research, University of Otago, Dunedin, New Zealand

${ }^{2}$ School of Rehabilitation Science, College of Medicine, University of Saskatchewan, Saskatoon, Saskatchewan, Canada

${ }^{3}$ Dunedin School of Medicine, University of Otago, Dunedin, New Zealand

Correspondence to Dr Daniel Cury Ribeiro; daniel.ribeiro@otago.ac.nz

\begin{abstract}
Introduction Low back pain is the greatest cause of years lived with disability worldwide and is linked with high societal and economic burden. Neuromuscular control impairments are a common clinical presentation in patients with non-specific low back pain. Musculoskeletal physiotherapists commonly use feedback as a part of the management of low back disorders. This systematic review will aim to assess the effectiveness of extrinsic biofeedback for reducing pain, disability and recurrence of pain in patients with non-specific low back pain. Methods and analysis Systematic searches will be performed in CINAHL, Embase, Medline, Psyclnfo, Scopus and Web of Science. We will include randomised controlled trial studies, if the study recruited patients with nonspecific low back pain; compared extrinsic feedback versus either placebo or control; another intervention; or in addition to an intervention versus that intervention alone; and have used pain, disability scores or low back pain recurrence as outcome measures. We will exclude studies with designs other than randomised controlled trials. We will assess the risk of bias within included studies using the PEDro scale, and the strength of evidence using the Grades of Recommendation, Assessment, Development and Evaluation approach.

Ethics and dissemination Ethical approval and patient consent are not required since this is a systematic review based on published studies. The results of this study will be published in an international peer-reviewed journal. PROSPERO registration number CRD42017077888
\end{abstract}

\section{INTRODUCTION}

Low back pain (LBP) is the most common musculoskeletal disorder, and the leading cause of years lived with disability. ${ }^{12}$ The accepted clinical subcategory of non-specific LBP is a multifactorial disorder and defined as "pain and discomfort, localised below the costal margin and above the inferior gluteal folds, with or without referred leg pain' ${ }^{3}$ The 1-month prevalence of LBP is $23.2 \%$. $^{2}$ Patients with non-specific LBP commonly present with slow recovery and may not fully recover within 12 months since first episode of LBP. ${ }^{4}$

Non-specific LBP is influenced by biological, psychological and social factors. ${ }^{5}$ These

\section{Strengths and limitations of this study}

- Comprehensive and exhaustive search for relevant studies from several databases.

- This review is limited to evidence from randomised controlled trials.

- No language restrictions will be imposed.

- The number of articles identified and included in this review may prevent us from conducting meta-analysis which might limit the conclusions that can be drawn from our findings.

three factors interact and impact on symptoms onset, recovery and clinical outcomes of patients with LBP. ${ }^{6}$ Biological factors include impaired motor control, ${ }^{7}$ delayed muscle activity of lumbopelvic muscles ${ }^{8}$ and reduced postural strategies for maintaining balance. ${ }^{9}$ Psychological factors (eg, fear avoidance, negative beliefs and depressive symptoms) may hinder recovery ${ }^{10}$ and may be associated with higher pain levels. ${ }^{11}$ Social factors such as family environment, socioeconomic and educational status and religion may modulate pain reported by patients with LBP. ${ }^{12}$

Management of non-specific LBP commonly focuses on education, pain control ${ }^{13}$ and exercise therapy targeting neuromuscular impairments. ${ }^{14}$ When targeting neuromuscular impairments, musculoskeletal physiotherapists may use extrinsic feedback to help patients improve movement control and awareness of lumbopelvic muscles. ${ }^{15}$ Extrinsic feedback can be defined as any form of information provided to the patient that originates from an external source (eg, mirror, pressure biofeedback, tactile or verbal input by the clinician). ${ }^{16} 17$ The way extrinsic feedback is provided to patients can hinder or improve motor control. ${ }^{18}$ Our previous review assessed how extrinsic feedback was provided to patients in trials and found that the majority of the studies did not adopt ideal forms of feedback provision. ${ }^{17}$ 
Table 1 Description of content and timing characteristics for extrinsic feedback provision

\begin{tabular}{|c|c|c|}
\hline Extrinsic feedback & Description & $\begin{array}{l}\text { Ideal/not } \\
\text { ideal } \\
\text { id } 2021^{2}\end{array}$ \\
\hline \multicolumn{3}{|l|}{ Content characteristics } \\
\hline Programme feedback & Feedback about general pattern of movement being executed. & Ideal \\
\hline Parameter feedback & $\begin{array}{l}\text { Feedback about specific components (eg, one element of the } \\
\text { whole movement) of movement being executed. }\end{array}$ & Not ideal \\
\hline Average feedback & $\begin{array}{l}\text { Mean score (based on error or performance score) calculated after } \\
\text { a number of attempts/trials are executed. }\end{array}$ & Ideal \\
\hline Summary feedback & $\begin{array}{l}\text { Qualitative feedback is provided after a number of attempts/trials } \\
\text { are executed. }\end{array}$ & Ideal \\
\hline Bandwidth (error magnitude) & $\begin{array}{l}\text { The amount of movement error that adopted a priori, and used to } \\
\text { provide feedback to patient. The bandwidth is used to distinguish } \\
\text { between successful and unsuccessful trials. }\end{array}$ & Ideal \\
\hline Internal focus of attention & $\begin{array}{l}\text { Feedback focused on body movement characteristics (eg, joint } \\
\text { angle). }\end{array}$ & Not ideal \\
\hline External focus of attention & Feedback focused on the effect of the movement. & Ideal \\
\hline \multicolumn{3}{|l|}{ Timing characteristics } \\
\hline Concurrent feedback & $\begin{array}{l}\text { Feedback provided to patient at the same time that the movement } \\
\text { is being executed. }\end{array}$ & Not ideal \\
\hline Terminal feedback & Feedback provided to patient after the movement was executed. & Not ideal \\
\hline Immediate & $\begin{array}{l}\text { Feedback provided to patient immediately after the movement } \\
\text { was executed. }\end{array}$ & Not ideal \\
\hline Delayed & $\begin{array}{l}\text { Feedback provided to patient after a period (eg, seconds or } \\
\text { minutes) of the movement was executed. }\end{array}$ & Ideal \\
\hline \multicolumn{3}{|l|}{ Frequency } \\
\hline Constant & Feedback provided at every trial. & Not ideal \\
\hline Reduced & Feedback provided for a fraction of trials (eg, $40 \%$ of trials). & Ideal \\
\hline Self-controlled & Feedback provided only when patient requests it. & Ideal \\
\hline
\end{tabular}

There are different ways of providing extrinsic feedback, and these can be grouped into two categories in relation to their content and timing characteristics. ${ }^{16} 1719$ Content characteristics refer to the focus of the intervention. ${ }^{1820}$ For example, how feedback is presented to patient (eg, absolute scores, average scores, general feedback about how movement is being and how it should be performed). ${ }^{1820}$ Timing characteristics refer to when the feedback is provided to patients (eg, simultaneously to exercise execution, after the exercise has been executed, or provided when the patient requests this information). ${ }^{18} 2021$ According to previous research on 'motor control and learning', there are some content and timing characteristics that will enhance/optimise motor control. ${ }^{182021}$ We present a description for content and timing characteristics of extrinsic feedback in table 1 .

A recent review reported that extrinsic feedback combined with physiotherapy compared with physiotherapy intervention alone was superior for pain reduction, but was no different to physiotherapy intervention alone for disability levels in patients with neck pain. ${ }^{19}$ Currently, the effectiveness of extrinsic feedback for the management of patients with non-specific LBP is unknown. The aim of the proposed systematic review is to assess the effectiveness of extrinsic biofeedback for reducing pain, disability and recurrence of pain in patients with non-specific LBP.

\section{METHODS}

Patient and public involvement

Patients and/or public were not involved in the development of this research project.

\section{Design}

This study will be a systematic review with meta-analysis, if data allow, and will be based on the Preferred Reporting items for systematic Reviews and Meta-Analyses Protocols checklist. $^{22}$

\section{Types of studies}

We will include randomised controlled trials, and exclude narrative reviews, systematic reviews, non-randomised clinical trials, cross-sectional studies and observational studies. We will not impose language restrictions. 


\section{Types of participants}

Studies must have recruited symptomatic individuals with non-specific LBP (acute, subacute or chronic), aged between 18 and 65 years. We will exclude studies involving patients with specific or systematic diseases (eg, tumours, cauda equina syndrome, fracture, inflammatory arthropathy).

\section{Types of interventions}

Any interventions that include the use of any form of extrinsic biofeedback (eg, verbal, tactile, pressure biofeedback, electromyography feedback, body positional biofeedback or other types of feedback) will be included in this review. We will exclude studies focusing on behavioural feedback or ergonomic training. Studies must include at least one of the following comparators:

- Extrinsic feedback versus placebo or control.

- Extrinsic feedback versus another form of intervention.

- Extrinsic feedback+interventionversus intervention alone.

\section{Types of outcome measures}

We will consider the following primary outcome measures: (1) pain must be measured with Visual Analogue Scale, Numeric Pain Scale or any other validated instrument; (2) disability levels must be measured with validated instruments (eg, Oswestry, Quebec Back Pain Disability Scale, the Roland-Morris Disability Questionnaire); (3) recurrence of LBP (as reported by patients or assessed by a clinical researcher). We will consider motor performance tests as secondary outcomes. Motor performance must be assessed using a form of biofeedback or electromyography pre-intervention and postintervention.

\section{Searches}

Systematic searches will be performed in the following databases from the inception: CINAHL, Embase, Medline, PsycInfo, Scopus and Web of Science. Search strategy is presented in table 2. This search strategy was developed in consultation with a health sciences librarian, tested and used for a review previously conducted by our research team. ${ }^{17}$

After the exclusion of duplicates, two independent reviewers will conduct title, abstract and full-text screening against inclusion criteria. If any disagreement persists, a third reviewer will adjudicate. The reference lists of included studies will be screened for additional relevant studies.

\section{Data extraction and management}

Data will be extracted by two reviewers independently and compared. If any disagreements persist and cannot be resolved by consensus, a third reviewer will be consulted. We will extract the following data: authors, year of publication, country of origin, study design, study purpose, experimental and comparison interventions and their characteristics, number of participants in each group and their characteristics, frequency of the interventions (if applicable), follow-up intervals (if applicable), drop-out rates, outcomes measures, main findings and authors' conflict of interest.

\begin{tabular}{|c|c|}
\hline Database & Search strategy \\
\hline \multirow[t]{3}{*}{ CINAHL } & 1 Low back pain \\
\hline & $\begin{array}{l}2 \text { Feedback OR Biofeedback OR } \\
\text { Extrinsic feedback }\end{array}$ \\
\hline & 1 AND 2 \\
\hline \multirow[t]{3}{*}{ Embase (keyword) } & 1 Low back pain OR backache \\
\hline & 2 Feedback system \\
\hline & 1 AND 2 \\
\hline \multirow[t]{3}{*}{ Medline (Ovid) } & 1 Low back pain \\
\hline & $\begin{array}{l}2 \text { Feedback OR Feedback, Sensory OR } \\
\text { Biofeedback, Psychology }\end{array}$ \\
\hline & 1 AND 2 \\
\hline \multirow[t]{3}{*}{ Psyclnfo (Ovid) } & 1 Back pain \\
\hline & $\begin{array}{l}2 \text { Biofeedback training OR biofeedback } \\
\text { OR feedback }\end{array}$ \\
\hline & 1 AND 2 \\
\hline \multirow[t]{3}{*}{ Scopus } & 1 Back pain \\
\hline & 2 Biofeedback OR feedback \\
\hline & 1 AND 2 \\
\hline \multirow[t]{3}{*}{ Web of Science } & 1 Back pain \\
\hline & 2 Biofeedback OR feedback \\
\hline & 1 AND 2 \\
\hline
\end{tabular}

Risk of bias within included studies and quality of evidence assessment

The risk of bias within included studies will be assessed using the PEDro scale. Reporting of the following aspects will be assessed: eligibility criteria, random allocation, concealed allocation, similarity between groups at baseline, blinding of subjects, therapists and assessors, attrition rate $<15 \%$, analysis by intention to treat, between-group comparison, and both point estimate and variability measures.

\section{Data synthesis and analysis}

If possible, we will use RevMan statistical software V.5.3 (The Nordic Cochrane Centre) for conducting the meta-analysis using a random-effects model. For the purpose of this review, outcome measures will be categorised into the following based on time points of assessment: immediate (within 2 weeks of the intervention delivery), short-term (2-13 weeks after intervention delivery), medium-term (14-50 weeks after intervention delivery) and long-term effects (51 or more weeks after intervention delivery).

For continuous data, we will calculate the mean difference (MD), and 95\% confidence intervals (CI) if outcome measure scales are the same. In the case of different outcome measure scales, we will calculate the standardized mean difference (SMD) and 95\% CI. For the purpose of this review, the effect size will be categorised as: small=SMD ranging from 0 to 0.2 ; medium $=\mathrm{SMD}$ 
ranging from $>0.2$ to $<0.5$; and large $=\mathrm{SMD}$ ranging from $>0.5$ to 0.8 . $^{2324}$

We will assess heterogeneity using $\mathrm{I}^{2}$ statistics ${ }^{25} 26$ and will consider heterogeneity to be substantial if $\mathrm{I}^{2}$ ranges from $50 \%$ to $90 \% .{ }^{27}$ Sensitivity analyses will be conducted by the quality of studies and by the length of follow-up. If possible, we will conduct subgroup analysis based on the type of the disorder (ie, acute, subacute or chronic non-specific LBP).

The strength of evidence will be assessed using the Grades of Recommendation, Assessment, Development and Evaluation (GRADE) approach. ${ }^{28}$ The GRADE approach uses four quality levels: high, moderate, low and very low. The strength of evidence will be downgraded by one level according to the following criteria: (1) limitations in the design and implementation of available studies suggesting risk of bias, (2) indirectness of evidence, (3) unexplained heterogeneity or inconsistency, (4) imprecision of results and (5) high probability of publication bias.

If meta-analysis is not possible, we will present a narrative synthesis of the findings. In this case, quantitative findings for each study will be descriptively reported and summarised.

\section{ETHICS AND DISSEMINATION}

This systematic review has been prospectively registered at the PROSPERO (CRD42017077888). The results of this review will be published in an international peer-reviewed journal.

Acknowledgements The authors thank Mr Richard German (Faculty Librarian) in assisting with the electronic search strategy for this review, and the financial support from the Centrefor Health, Activity and Rehabilitation Research (CHARR), School ofPhysiotherapy - University of Otago.

Contributors DCR is the leading researcher and was responsible for conceiving this study, designing the protocol, preparing the search strategy and coauthoring the first draft of this manuscript. He has accepted the final version of the manuscript for submission. AKM contributed to the design of the review protocol, preparing the search strategy and coauthoring the first draft of this manuscript. JHA has contributed to the design of the review protocol and to the manuscript. SM contributed to the design of the review protocol and to the manuscript. DCR (principal investigator), JHA (coinvestigator) and SM (coinvestigator) secured the Health Research Council Emerging Researcher First Grant (15/527). All authors have contributed to the conception and design of the study protocol, development of the search strategy, the establishment of the inclusion and exclusion criteria, data extraction criteria, analyses and interpretation, and accepted the final version of the manuscript for submission.

Funding DCR (principal investigator), JHA (coinvestigator) and SM (coinvestigator) are supported by the Health Research Council Emerging Researcher First Grant (15/527).

Competing interests None declared.

Patient consent Not required.

Ethics approval Not required.

Provenance and peer review Not commissioned; externally peer reviewed.

Open Access This is an Open Access article distributed in accordance with the Creative Commons Attribution Non Commercial (CC BY-NC 4.0) license, which permits others to distribute, remix, adapt, build upon this work non-commercially, and license their derivative works on different terms, provided the original work is properly cited and the use is non-commercial. See: http://creativecommons.org/ licenses/by-nc/4.0/

(C) Article author(s) (or their employer(s) unless otherwise stated in the text of the article) 2018. All rights reserved. No commercial use is permitted unless otherwise expressly granted.

\section{REFERENCES}

1. Hoy D, March L, Brooks P, et al. The global burden of low back pain: estimates from the Global Burden of Disease 2010 study. Ann Rheum Dis 2014;73:968-74.

2. Hoy D, Bain C, Williams G, et al. A systematic review of the global prevalence of low back pain. Arthritis Rheum 2012;64:2028-37.

3. Airaksinen O, Brox JI, Cedraschi C, et al. Chapter 4. European guidelines for the management of chronic nonspecific low back pain. Eur Spine J 2006;15(Suppl 2):S192-300.

4. Henschke N, Maher CG, Refshauge KM, et al. Prognosis in patients with recent onset low back pain in Australian primary care: inception cohort study. BMJ 2008;337:a171.

5. O'Sullivan $\mathrm{P}$, Caneiro JP, O'Keeffe $\mathrm{M}$, et al. Unraveling the complexity of low back pain. J Orthop Sports Phys Ther 2016;46:932-7.

6. Feyer AM, Herbison P, Williamson AM, et al. The role of physical and psychological factors in occupational low back pain: a prospective cohort study. Occup Environ Med 2000;57:116-20.

7. Tsao H, Tucker KJ, Hodges PW. Changes in excitability of corticomotor inputs to the trunk muscles during experimentallyinduced acute low back pain. Neuroscience 2011;181:127-33.

8. Hodges PW, Moseley GL, Gabrielsson A, et al. Experimental muscle pain changes feedforward postural responses of the trunk muscles. Exp Brain Res 2003;151:262-71.

9. Caffaro RR, França FJ, Burke TN, et al. Postural control in individuals with and without non-specific chronic low back pain: a preliminary case-control study. Eur Spine J 2014;23:807-13.

10. George SZ, Beneciuk JM. Psychological predictors of recovery from low back pain: a prospective study. BMC Musculoskelet Disord 2015;16:49

11. Urquhart DM, Bell RJ, Cicuttini FM, et al. Negative beliefs about low back pain are associated with high pain intensity and high level disability in community-based women. BMC Musculoskelet Disord 2008:9:148.

12. Rodrigues-de-Souza DP, Palacios-Ceña D, Moro-Gutiérrez L, et al. Socio-Cultural factors and experience of chronic low back pain: a Spanish and Brazilian Patients' perspective. A qualitative study. PLoS One 2016;11:e0159554.

13. Maher $\mathrm{C}$, Underwood M, Buchbinder R. Non-specific low back pain. Lancet 2017;389:736-47.

14. Falla D, Hodges PW. Individualized exercise interventions for spinal pain. Exerc Sport Sci Rev 2017;45:105-15.

15. Hides JA, Stanton WR, Wilson SJ, et al. Retraining motor control of abdominal muscles among elite cricketers with low back pain. Scand J Med Sci Sports 2010;20:834-42.

16. Ribeiro DC, Sole G, Abbott JH, et al. A rationale for the provision of extrinsic feedback towards management of low back pain. Man Ther 2011;16:301-5

17. Ribeiro DC, Sole G, Abbott JH, et al. Extrinsic feedback and management of low back pain: a critical review of the literature. Man Ther 2011;16:231-9.

18. Magill RA. Motor learning and control: concepts and applications. 8th Edn. London: McGraw-Hill, 2007.

19. Araujo FX, Scholl Schell M, Ribeiro DC. Effectiveness of physiotherapy interventions plus extrinsic feedback for neck disorders: a systematic review with meta-analysis. Musculoskelet Sci Pract 2017;29:132-43.

20. Schmidt R, Wrisberg C. Motor learning and performance: a situationbased learning approach. Champaign, IL: Human Kinetics, 2008.

21. van Vliet PM, Wulf G. Extrinsic feedback for motor learning after stroke: what is the evidence? Disabil Rehabil 2006;28:831-40.

22. Moher D, Shamseer L, Clarke M, et al. Preferred reporting items for systematic review and meta-analysis protocols (PRISMA-P) 2015 statement. Syst Rev 2015;4:1.

23. Cohen J. Statistical power analysis for the behavioral sciences. 2nd Edn. Hillsdale, NJ: Lawrence Erlbaum, 1988.

24. Higgins JP, Green S. Cochrane Handbook for Systematic Reviews for Interventions Version 5.0.2. The Cochrane Collaboration 2009. updated Sep 2009.

25. Huedo-Medina TB, Sánchez-Meca J, Marín-Martínez F, et al. Assessing heterogeneity in meta-analysis: $\mathrm{Q}$ statistic or $\mathrm{I} 2$ index? Psychol Methods 2006;11:193-206.

26. Deeks JJ, Higgins JP, Altman DG. Analysing data and undertaking meta-analyses. In: Deeks JJ, Green S, eds. Cochrane handbook for systematic reviews of interventions version 5.1.0, 2011.

27. Higgins JP, Thompson SG. Quantifying heterogeneity in a metaanalysis. Stat Med 2002;21:1539-58.

28. Guyatt GH, Oxman AD, Vist GE, et al. GRADE: an emerging consensus on rating quality of evidence and strength of recommendations. BMJ 2008;336:924-6. 